\title{
HIGHLIGHTS
}

\section{PANCREATIC CANCER}

\section{Gemcitabine confirmed as the first-line therapy for pancreatic cancer}

Researchers have found that a combination of 5-fluorouracil, folinic acid and cisplatin (LV5FU2-CDDP) does not improve overall or progression-free survival when used as a first-line therapy instead of gemcitabine in patients with pancreatic cancer.

Gemcitabine is regarded as the gold standard first-line therapy for patients with pancreatic cancer, but treatment options are limited and patients are unlikely to survive for $>5$ years. Many studies have tried to increase the efficacy of gemcitabine treatment, but so far none of these regimens has improved survival.

In a multicenter phase III trial, 202 patients were randomly allocated to receive either LV5FU2-CDDP then gemcitabine after progression or the reverse sequence. After a median follow-up of 44 months there was no significant difference in survival between the two treatment arms.
However, the authors found that the patients tolerated gemcitabine as a firstline treatment better than LV5FU2-CDDP. Indeed, many patients who received LV5FU2-CDDP had severe adverse effects, which resulted in reduced patient compliance. These findings suggest that gemcitabine should remain as the standard first-line therapy for patients with pancreatic cancer.

Currently, there is no standard therapy for patients whose cancer progresses after receiving gemcitabine. The authors suggest that a platinum-based compound, such as oxaliplatin, would be a suitable second-line treatment in these patients.

\section{Claire Greenhill}

Original article Dahan, L. et al. Combination 5-fluorouracil, folinic acid and cisplatin (LV5FU2-CDDP) followed by gemcitabine or the reverse sequence in metastatic pancreatic cancer: final results of a randomised strategic phase III trial (FFCD 0301). Gut 59, 1527-1534 (2010) 